\title{
Development of mechanisms for innovative projects management based on their identification and results forecast in the risk environment
}

\author{
Ella Okolelova ${ }^{1}$, Natalya Trukhina ${ }^{2 *}$ \\ ${ }^{1}$ Voronezh State Technical University, Russia 394026, Moscow Avenue, Voronezh 14, Russia \\ ${ }^{2}$ Moscow State University of Civil Engineering, 129337, Yaroslavskoye Shosse 26, Moscow, Russia
}

\begin{abstract}
The article presents the essential characteristics of innovative projects and classifies them by grade of novelty and types of innovative products to be created. The classification features allow us to identify the categories of tangible innovative products and innovative technologies. The article contains the main challenges in evaluating, forecasting and managing innovative projects in the risk environment. An innovative project is considered as a complex of possible implementations, i.e. as a random function. The investors' goal is to determine the most likely outcome of project implementation for making further decisions.
\end{abstract}

\section{Introduction}

The innovative projects vary in terms of functional purpose, tasks and characteristics. The functional purpose of an innovative project also varies on the basis of its "novelty carrier" extent, i.e. whether the product underlying the economic feasibility study of an innovative project is fundamentally new for the market, or the consumer sees improved characteristics of an existing product that allow to extend the range of the functions performed and tasks that were fulfilled previously.

Before considering the mechanisms for management of an innovative project, it is needed to identify the subject of management as an innovative project, determine the main evaluation criteria, systematize the project characteristics, identify possible risks, evaluate the development strategies, etc.

To determine the characteristics of an innovative project, first, it is needed to evaluate the grade of novelty of the product, the implementation of which it is aimed at. Therefore, it is required to evaluate the novelty of the original product and determine to which extent a new product differs from those previously existing in the market, which new or additional tasks it allows to fulfill from the user's point of view, to which extent its characteristics meet the consumer preferences of the market, etc.

\footnotetext{
* Corresponding author ntruhina@list.ru
} 


\section{Classification of innovative products}

The following three innovative levels which determine the grade of novelty and the functional and qualitative level of an innovative project can be identified:

- absolutely new products (grade of novelty I);

- substitute products (grade of novelty II);

- analog products (grade of novelty III).

Absolutely new products pertaining to the first category are the products which do not have any analogs on the market and do not substitute any existing products, are designed to perform new functions and fulfill new tasks, have significant qualitative differences from the products positioned on the market, and are designed to perform fundamentally new functions.

Substitute products are characterized by a significant improvement in technical, qualitative and other characteristics and are designed to provide a substitute on the market of obsolete goods not meeting the up-to-date consumer requirements.

Analog products enter the market in parallel with the related products and perform the same functions, but have a significant difference in their qualitative characteristics and extend significantly the range of tasks to fulfill.

Table 1 Classification characteristic of innovative products based on grade of novelty

\begin{tabular}{|l|l|l|}
\hline \multicolumn{1}{|c|}{$\begin{array}{c}\text { Grade of } \\
\text { novelty }\end{array}$} & \multicolumn{1}{|c|}{ Functions } & \multicolumn{1}{|c|}{ Tasks } \\
\hline $\begin{array}{l}\text { Absolutely } \\
\text { new products }\end{array}$ & $\begin{array}{l}\text { Fundamentally new functions, } \\
\text { new purpose of good. }\end{array}$ & $\begin{array}{l}\text { New tasks which were either } \\
\text { impossible or quite hard to fulfill } \\
\text { before }\end{array}$ \\
\hline $\begin{array}{l}\text { Substitute } \\
\text { products }\end{array}$ & $\begin{array}{l}\text { The same functions, extension } \\
\text { of the range of functions and } \\
\text { improvement of the basic } \\
\text { functions are possible }\end{array}$ & $\begin{array}{l}\text { Fulfillment of the basic tasks, } \\
\text { extension of the range of tasks, } \\
\text { including fulfillment of new ones. }\end{array}$ \\
\hline $\begin{array}{l}\text { Analog } \\
\text { products }\end{array}$ & $\begin{array}{l}\text { The functions remain the same, } \\
\text { addition of new functions is } \\
\text { possible }\end{array}$ & $\begin{array}{l}\text { Fulfillment of the basic tasks with } \\
\text { possible addition of new ones }\end{array}$ \\
\hline
\end{tabular}

An innovative product can be represented by new goods or by a new material used for creating the final market product, having qualitative indicators and characteristics changed significantly. The innovative products also include intangible objects which represent development of new technologies, building of a production management system, formation of a product quality management system, etc.

The category of intangible innovative products can include fundamentally new intercorporate systems, connections and relations aimed at the improvement of the mechanisms of economic interaction, development of efficient communications and mediation connections.

Therefore, the innovative products can be differentiated by their essential content as follows:

- tangible objects;

- technologies;

- structural and management systems and relations;

- organizational and corporate systems and relations.

\section{Formation of innovative solutions}


The common purpose of creating an innovative product of any of the presented categories is profit-making, i.e. presence of business effect. Other types of effects are also possible, for example, social, environmental, etc. determined based on the purposes of implementation of the innovative project. The essential content of the classification features of tangible innovative products is shown on Fig. 1.

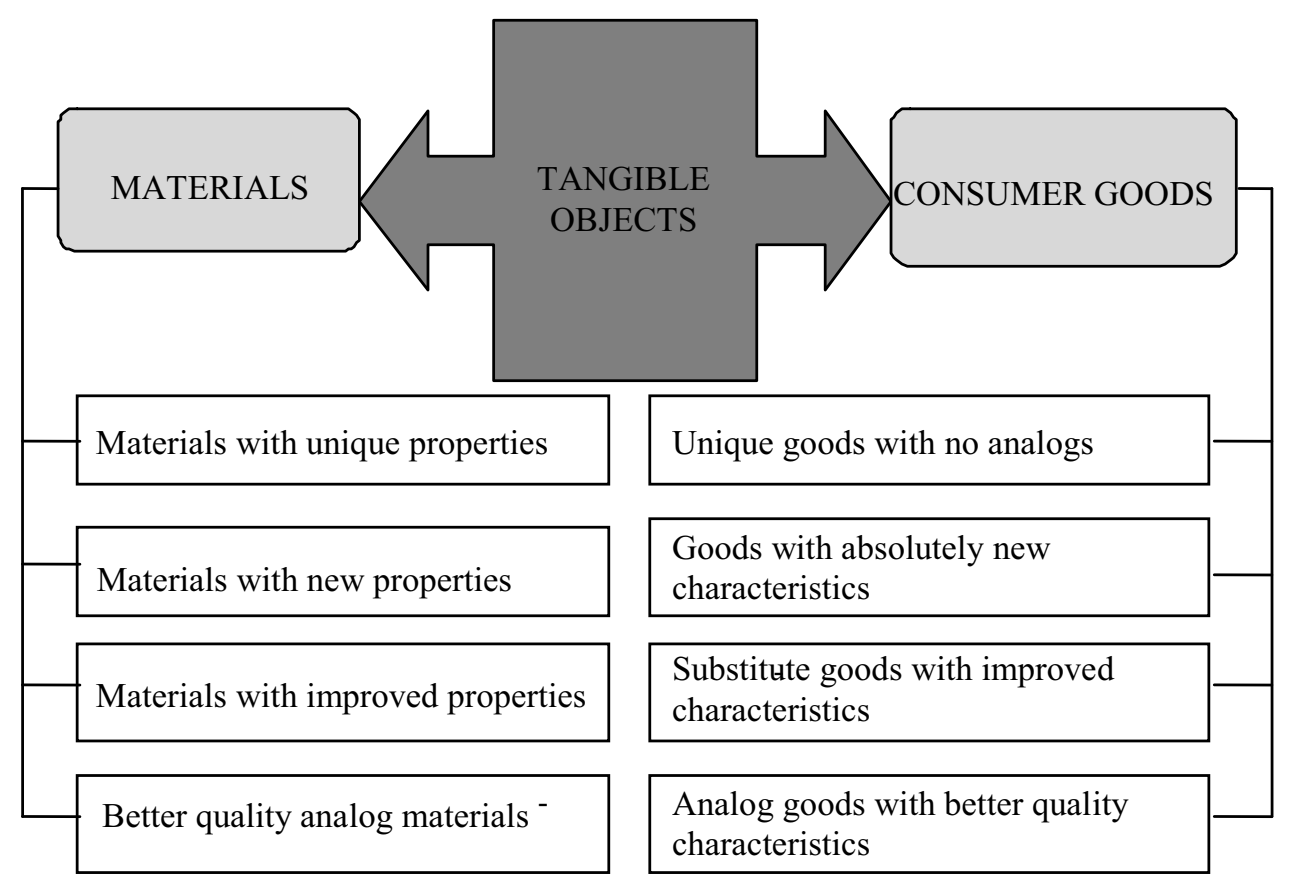

Fig. 1.The essential content of the classification features of tangible innovative products.

Another type of innovative product to which implementation can be aimed at are technologies.

The following classification of innovative technologies is proposed based on the purposes of creation of innovative technologies:

- manufacturing, aimed at creating a tangible product;

- information technologies;

- information and communication technologies.

- organization and management technologies;

The grade of innovativeness and creativity of technologies is determined by the novelties or improvements they carry. An innovative technology can have features of a fundamentally new, newly applied technology, if it contains innovativeness as to the application of materials and resources, technical performance, use of improved equipment, application of new manufacturing flowcharts, methods, processes, etc., as shown on Fig. 2. 


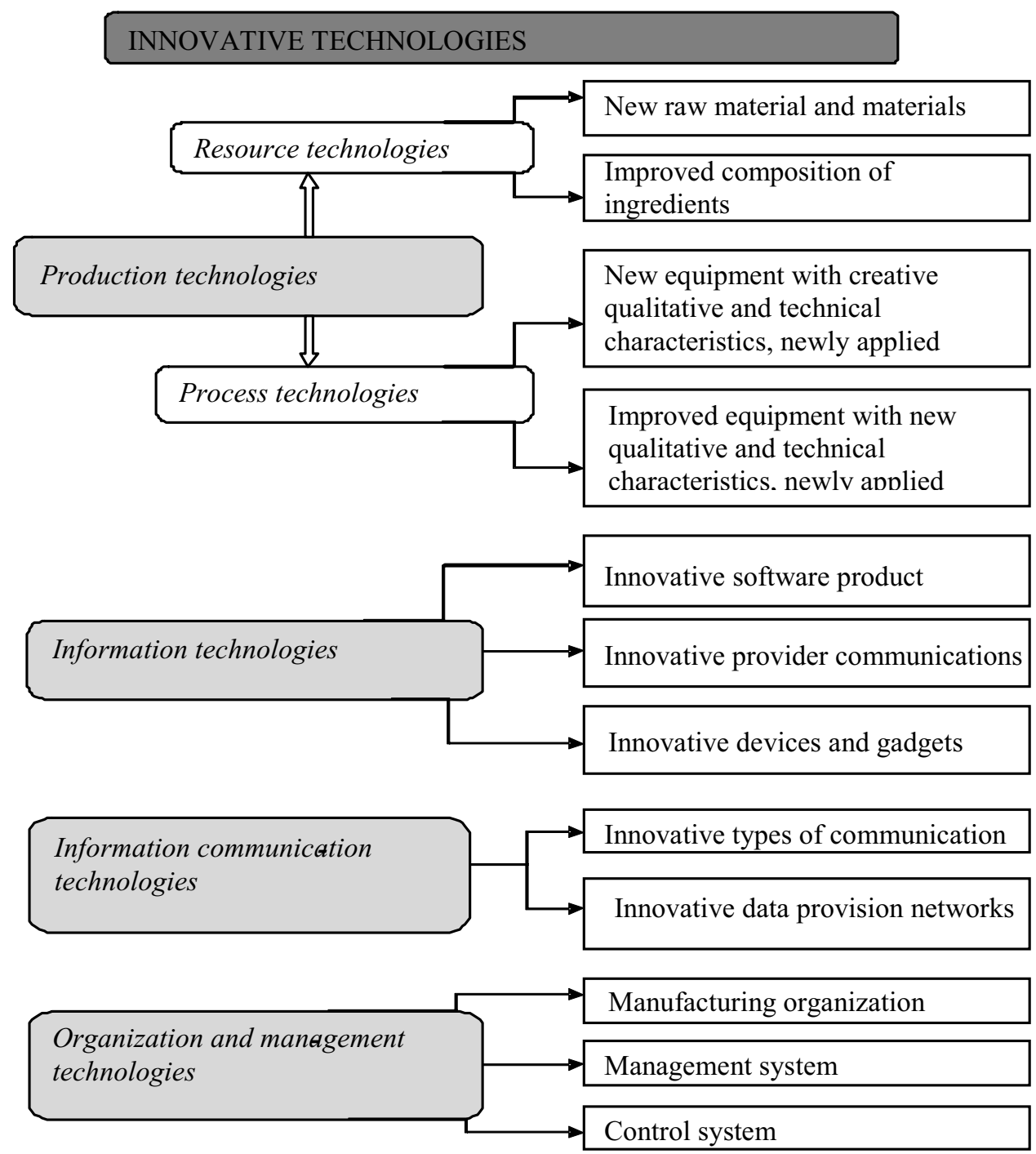

Fig. 2. Content of the classification features of innovative technologies.

It is especially shown in manufacturing technologies. Thus, a technology can have fundamentally new differences in terms of using raw material components, ingredients proportionand include new components, etc. This type of improvement of manufacturing technologies is defined as resource technologies.

The resource technologies imply significant change in the raw materials for the manufacturing of the final product.

The machines and equipment for manufacturing the final product are also subject to improvement. This type is defined as process technologies. 
Contentwise, the information technologies can be oriented for development of a new software product, can be based on innovative provider communications, as well as on improved and fundamentally new devices and gadgets.

In terms of innovation, the information and communication technologies represent the creation of new possibilities for provision and receipt of data, development of new types of communication, etc.

The innovative organization and management technologies can include creative structural changes in the manufacturing process management, changes in the control functions, management systems of both the manufacturing and separate processes.

This classification category represents innovative enterprise management mechanisms distinguished by the building of fundamentally new structural communicative networks proving not only the presence a new approach to the manufacturing process management and control functions, but its efficiency as well.

The methodology for project identification allows to determine possible strategies of its development, assess the risks of the project and forecast the economic effect of its implementation.

The innovative projects, generally, present high risks. It is quite difficult to forecast their development, their implementation in the manufacturing process and the assessment of efficiency of their implementation. Risk is inevitable when implementing innovative projects under uncertainty. Therefore, it is preferable to make decisions while having certain safety reserve, the essence of which is to formulate arguments allowing to affirm with reasonable certainty that the investor will not incur any critical losses, even if the key parameters were set too high (too law) during the forecast.

Regardless of the forecasted project profitability value (for example, the volume of implementation and the value of income), there will always be different factors affecting the accuracy of the final forecasts. An innovative project can be considered as a complex of possible implementations, i.e. as a random function.

Therefore, speaking of an innovative project, we can affirm that there is a functional dependency of the economic effect on a series of governing parameters over time. Based on the actual practical conditions of the environment, we will define an innovative project as a random function of a nonrandom argument $t$, which is a random value at each fixed value of the argument.

If we present an innovative project as a dynamic model, it is possible to assume that such model will be stable under certain conditions depending, for example, on the value of financial investments, forecasted level of income from the implementation of the project, inflation, etc.

In case of change in one or more of the above-mentioned parameters, the dynamic system can move to a whole new level, i.e. bifurcation changes arise [4].

We define an innovative project as a random function, i.e. a function of the nonrandom argument $t$, which is a random value at each fixed value of the argument. If the value $P$ of the expected income from the implementation of the project is a random value, then the function of profitability of the investment project is also random:

$$
F(t)=S(P ; t) \text {. }
$$

Let us consider the model of a dynamic system with random perturbations. Such models describe a wide class of phenomena of the real world, including economic processes, and represent systems of ordinary stochastic differential equations of the $\mathrm{n}^{\text {th }}$ order complemented by the corresponding initial conditions which can always be transformed into an equivalent system of $\mathrm{n}$ differential equations of the 1 st order as follows:

$$
y_{v}(t)=Y_{v}\left(y_{1}, y_{2}, \ldots, y_{n}\right), \quad v=1,2, \ldots n \text {. }
$$


Here, $y_{v}(t)$ are any dependent variables related with "motion", i.e. with temporary (dynamic) course of the process.

The system motion which we will call an unperturbed motion, as opposed to another motion which we will define as perturbed motion $y_{v}(t)$ [1], will correspond to a partial solution $f_{v}(t)$ of one of the system of equations (2)

$$
f_{v}(t)=Y_{v}\left(t_{1}, f_{1}, f_{2} \ldots, f_{n}\right), \quad v=1,2, \ldots n .
$$

The difference between the values of the perturbated $y_{v}(t)$ and nonperturbed $f_{v}(t)$ motions at each moment of time $t$ will be called perturbation $x_{v}(t)$ :

$$
y_{v}(t)-f_{v}(t)=x_{v}(t) \text {. }
$$

The nonperturbed motion $f_{v}(t)$ must satisfy the system of equations under the following conditions:

$$
\begin{aligned}
& \left|x_{v}\left(t=t_{0}\right)\right| \leq \delta ; \\
& \left|x_{v}\left(t>t_{0}\right)\right| \leq \varepsilon(\delta) ; \\
& \lim _{t \rightarrow \infty}\left|x_{v}(t)\right|=0 .
\end{aligned}
$$

An unperturbed motion is called stable, if for any small positive number $\delta>0$ another such number $\varepsilon(\delta)$ can be found, so that the inequality (5) could be carried out for all perturbed motions $y_{v}(t)$ for the initial moment of time $t=t_{0}$, and the inequality (6) would be true at all subsequent moments of time $t>t_{0}$. Otherwise, an unperturbed motion will be unstable [4].

The definition of the asymptotical stability is more rigorous than the previous definition. In case of an asymptotically stable motion perturbed at the initial moment of time $t=t_{0}$, the perturbations not only remain within the neighborhood of the initial condition $\varepsilon(\delta)$, as in case of normal stability, but, in addition, eventually fade to zero [4]. To formalize further consideration of the issue, let us introduce a series of concepts which will allow us to quantitatively characterize the perturbations of the mathematic models of the following type

$$
y=P_{n}(s) u,
$$

where $P_{n}(s)$ is a nominal transfer function [2]. The object with a mathematic model (8) will be stabilized by the regulator

$$
u=-K(s) y,
$$

where $K(s)$ is a transfer function of the system (8).

Suppose that the transfer function $K(s)$ of the regulator does not change in the process of functioning, and the transfer function $P_{n}(s)$ of the object is affected by nonparametric type unstructured perturbations. As the result of such impact, the regulator (9) will actually complete a different object and not the object with the model (8)

$$
y=P(s) u,
$$

the transfer function $P(s)$ of which will differ from the nominal one.

Let us determine the indicator $\Delta_{A}(s)$ of the absolute perturbation of the model (or the perturbation of the nominal transfer function $P_{n}(s)$ ).

$$
\Delta_{A}=P(s)-P_{n}(s) \text {. }
$$

Accordingly, we will call the following rational fraction a relative perturbation of the model or of the nominal transfer function: 


$$
\Delta_{0}=\left[P(s)-P_{n}(s)\right] P_{n}^{-1}(s) .
$$

The following formula will be considered a weighted relative perturbation of the model or of the nominal transfer matrix:

$$
\Delta_{p}(s)=\left[P(s)-P_{n}(s)\right]\left[P_{n}(s) W_{d}(s)\right]^{-1},
$$

where $W_{d}(s)$ is the prescribed weight fractional rational function.

The introduction of the weight function $W_{d}(s)$ in the determination of the perturbation (13) is conditioned by the amplitude frequency characteristics (AFCh) $A_{n}(\omega)=\left|P_{n}(\omega)\right|$ and $A(\omega)=|P(\omega)|$ for the nominal and the perturbed objects respectively. Let us introduce an admissible boundary of the nominal mathematic model perturbation, determining it by an upper bound (for each frequency) on the value of the relative change module of the AFCh by a positive number $\left|W_{d}(\omega)\right|$. In other words, the introduction of the function $W_{d}(s)$ determines the condition

$$
\left|\frac{P(\omega)|-| P_{n}(\omega) \mid}{P_{n}(\omega)}\right| \leq\left|W_{d}(\omega)\right|, \quad \forall \omega \in R^{1},
$$

prescribing the admissible "corridor" for AFCh variations of the actual (perturbed) object (8), which is shown on Fig. 2.

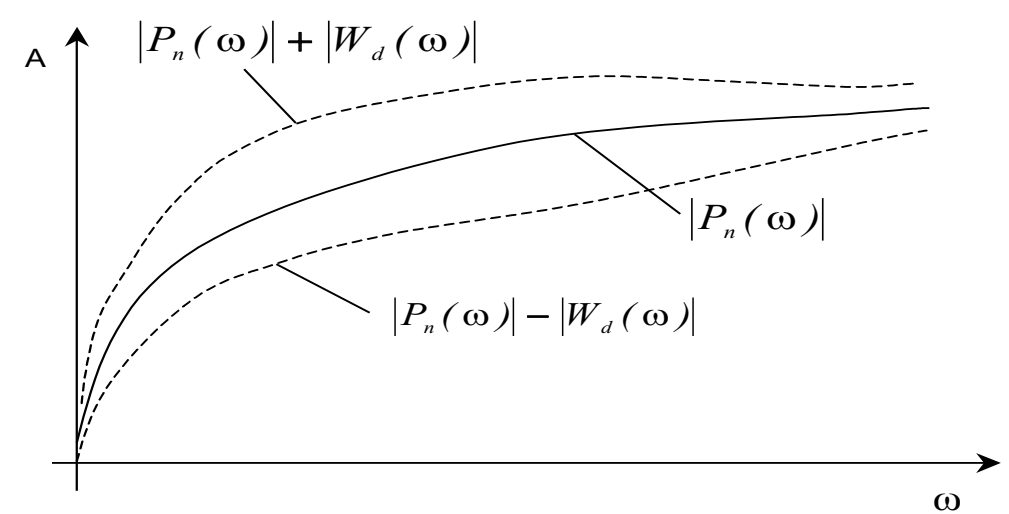

Fig. 3. An admissible "corridor" for AFCh variations of the actual perturbed abject

Therefore, the frequency function $\left|W_{d}(\omega)\right|$ is the relative width of the admissible corridor for the AFCh of the perturbed object. Note that it is not quite convenient to work analytically with the condition (14). Instead of it, we can use the relation of the following type

$$
\left|\frac{P(\omega)-P_{n}(\omega)}{P_{n}(\omega)}\right| \leq \mid W_{d}(\omega), \quad \forall \omega \in R^{1},
$$

which is stronger than (14), which results from the following inequality

$$
\left\|P(\omega)|-| P_{n}(\omega)\right\| \leq\left|P(\omega)-P_{n}(\omega)\right|, \quad \forall \omega \in R^{1},
$$

true for any two complex numbers $P$ and $P_{n}$. Therefore, carrying out (15) will cause carrying out (14). 


\section{Conclusion}

Therefore, it is possible to determine the "corridor" of the values of the forecasted parameters. Economically, these will be the limit values of the impact of both external and internal factors on the result of the investment project implementation. The instability conditions for an economic system can be determined quantitatively with the conditions of the tolerances, providing an opportunity to forecast the results of the process subject to nonparametric impact.

\section{References}

1. E.Yu. Okolelova, O.V. Kornitskaya, N.I. Trukhina, Management of Economic Systems scientific electronic journal 60 (2013)

2. N.I. Trukhina, I.I. Chernyshikhina, Proceedings of Moscow State University of Civil Engineering 9, 227-233 (2012)

3. E.Yu. Okolelova, Hannover, 96-101 (2016)

4. E.Yu. Okolelova, A.V. Shabanov, News of the Universities of the Black Earth Region T.1, 96-99 (2006)

5. I. Karakozova, Y. Prokhorova, MATEC Web of Conferences 07015, 73 (2016)

6. V.S. Morozov, N.N. Taskaeva, Journal of Internet Banking and Commerce 21 (4), 20 (2016)

7. G. Alexander, R. Anastacia, International Journal of Applied Engineering Research 10 (21), 42410-42411 (2015)

8. G. Alexander, R. Anastacia, International Journal of Applied Engineering Research 10 (21), 41828-41830 (2015)

9. G. Bubnov, B.Titarenko, S. Titov, R. Titarenko, Information Processing and Control Engineering 1 (2015) 